\title{
CALIDAD DE LA SEMILLA DE LÍNEAS E HÍBRIDOS DE SORGO SOMETIDOS A INTEMPERISMO ${ }^{1}$
}

\author{
Raúl Rodríguez², Fernando Narvaez ${ }^{3}$, Héctor Williams ${ }^{2}$
}

\section{RESUMEN}

Calidad de la semilla de líneas e híbridos de sorgo sometidos a intemperismo. En el Campo Experimental Río Bravo durante el ciclo otoño-invierno 1992-93, se evaluaron dos líneas mantenedores (susceptibles y tolerantes a deterioro de campo), cuatro líneas restauradoras y los ocho híbridos posibles de la cruza de estas líneas. Las pruebas se efectuaron con el grano bajo condiciones naturales de deterioro de campo, se cosechó el grano a los 0, 11, 22 y 33 días después de haber alcanzado la madurez fisiológica. Los resultados indicaron que la línea LRB-118B (tolerante) mostró mayor peso volumétrico y vigor que LRB-104B (susceptible), confiriéndose estas ventajas a los híbridos. Para peso de 1.000 semillas y germinación estándar no se encontraron diferencias significativas.

\begin{abstract}
Seed quality of lines and sorghum hybrids subjected to weathering. A study was carried out in the Rio Bravo Experimental Station during the 1992-93 fall-winter cycle to evaluate two maintainer lines (susceptible and tolerant to weathering), four restorer lines, and the eight hybrids resulting from crossing these lines. Trials were conducted on lines and hybrids exposed to weathering, and grains were harvested $0,11,22$, and 33 days after they were physiologically mature. Results indicated that the LRB-118B tolerant line showed greater weight/volume ratio and seeding vigor that the LRB-104B susceptible line, and conferred these advantages on its hybrids. Significant differences were not found for the weight of 1,000 seeds and standard germination.
\end{abstract}

\section{INTRODUCCIÓN}

El área más extensa sembrada con sorgo en México se ubica en el norte de Tamaulipas, donde en el año de 1994, se cosecharon 557.193 ha y se obtuvo una producción de 1.375.505 t de grano (SARH, 1994). En esta región la cosecha se realiza durante el mes de junio, coincidiendo frecuentemente con lluvias y alta humedad relativa del aire, las cuales no sólo reducen el rendimiento al provocar el inicio de la germinación del grano aun en la panoja, sino que favorecido por temperaturas altas crean un ambiente propicio para el desarrollo de los hongos. En algunas ocasiones debido a la extensa superficie sembrada y a la insuficiente cantidad de maquinaria agrícola (camiones y trilladoras), la cosecha se retarda en algunos lotes por varias semanas después de haber alcanzado el grano la madurez fisiológica, tal como ocurrió durante el ciclo agrícola otoñoinvierno 1992-93. Por otra parte no sólo es afectada la producción comercial, sino que debido a la importancia que representa en la región la producción de semilla certificada para siembra, ésta se suele deteriorar muy fácilmente en sus características tanto físicas como fisiológicas, llegando en algunos años a perderse casi en su totalidad (90\%), tal como ocurrió durante el ciclo otoño-invierno 1991-92.

La ocurrencia de la madurez fisiológica de la semilla puede ser considerada como un proceso positivo que consiste en un incremento en el tamaño de la semilla, acumulación de peso seco, desarrollo de las estructuras esenciales, una pérdida de humedad y un incremento en su viabilidad y vigor. Casi inmediatamente después de la madurez fisiológica, la semilla entra en su fase negativa caracterizada por una declinación en su viabilidad y vigor causada por: la respiración, alta temperatura y humedad, daño mecánico y el tiempo de exposición a las condiciones climáticas (Avila, et al., 1994, Rajanna y

1 Presentado en la XLIII Reunión Anual del PCCMCA en Panamá 1997.

2 Programa de Cultivos Industriales. Sorgo. SAGAR-INIFAP-CIRNE. Campo Experimental Río Bravo. Apartado Postal No.172. Río Bravo, Tam. C.P. 88900.

3 CCDTS-UAAAN. Buenavista, S altillo, Coah. México. 
Andrews, 1970). La conservación de la capacidad de gerrninación y de emergencia de la semilla dependerá del ulterior manejo que se le otorgue a ésta, después de alcanzar la madurez fisiológica, el cual es el punto de máxima calidad (Rivera y Gasca, 1994; Delouche, 1964).

Existe consenso de que los principales factores ambientales que reducen la capacidad de germinación de la semilla son la alta humedad relativa y temperatura del aire (Roberts, 1973). Las fluctuaciones bruscas de estas reducen drásticamente la calidad de la semilla (López; Molina; Carballo, 1994). La calidad de la semilla está basada en muchos factores siendo los mas importantes: germinación, pureza, sanidad, capacidad de almacenamiento y vigor (Bragonier, 1976).

El problema del deterioro del grano en el campo de sorgo en México no está bien documentado, sin embargo se percibe que es importante, ya que en la mayoría de las áreas productoras se presentan lluvias y alta humedad relativa del aire, cuando el cultivo se encuentra en las etapas de formación de grano y madurez fisiológica. Por lo anterior es conveniente que los programas de mejoramiento genético de sorgo del país, al formar nuevo germoplasma tomen en consideración este aspecto.

Durante el ciclo otoño-invierno 1990-91 dentro del lote de mejoramiento gen ético de sorgo del Campo Experimental Río Bravo, al llover continuamente durante un período de 10 días en la época de cosecha, se logró seleccionar visual mente a la línea LRB-118B como tolerante al intemperismo. Esta línea se seleccionó de LRB-104B (susceptible) progenitora del híbrido comercial RB-4000. El objetivo del presente estudio fue determinar la tolerancia de ambas líneas al intemperismo y comprobar si confieren resistencia a la progenie cuando se cruzan con diferentes líneas restauradoras.

La pérdida en peso volumétrico (PV) durante las cosechas retrasadas es comúnmente encontrado en la literatura y ésta se ha atribuido a los cambios en la forma del grano o arrugamiento de la capa superficial, debido a una reducción neta de su densidad o de su masa (Johnson, 1959). La reducción en el peso de 1.000 semillas ha sido atribuida a una pérdida en la masa del grano, debido a un aumento en la respiración, cuando éste se desarrolla en condiciones de alta humedad y temperatura (Woodstock; Furman; Leffler, 1985). La alta temperatura y humedad relativa del aire juegan un papel importante cuando se retrasa la cosecha en el norte de Tamaulipas, ya que se realiza durante la época más calurosa del año, que coincide regularmente con lluvias que incrementan la humedad relativa del aire y del grano.

\section{MATERIALES Y MÉTODOS}

Genotipos: Se evaluaron ocho híbridos producto de la cruza de dos líneas androestériles, LRB-104A (susceptible al deterioro del grano en el campo) y LRB118A (tolerante) con cuatro líneas restauradoras, seleccionadas de acuerdo a características contrastantes (Cuadro 1). La línea LRB-118B se formó de LRB-104B (progenitora del híbrido RB-4000), se utilizó el método de retrocruza hasta obtener la generación RC3F2, mientras se incorporaba el carácter color de planta canela de la línea M-60009B proporcionada por el programa de ICRISAT-LASIP. Se formaron dos líneas genéticamente muy similares pero diferentes en color de planta y tolerancia al deterioro del grano en el campo.

Las líneas restauradoras utilizadas fueron: LRB-25 progenitora del híbrido RB-4000 caracterizada por producir híbrido s con un potencial de rendimiento superior al promedio de los comerciales; Tx-430 en sus versiones isogénicas para color de planta roja y canela, en donde las plantas canela en general presentan menor cantidad de taninos en el grano; SC-I03 de grano color café es tolerante a pájaros y está asociada a alto contenido de taninos en el grano. Las líneas Tx-430R y SC103 provienen de la Universidad de Texas A\&M. El resto de las líneas fueron formadas en el Campo Experimental Río Bravo perteneciente al INIFAP. Tx-430 es progenitora de muchos híbridos comerciales de compañías privadas y de PRONASE (Cuadro 1).

Las dos líneas androestériles (A), dos mantenedores (B) y cuatro restauradoras (R) fueron sembradas el 8 de marzo de 1993 en el Campo Experimental Río Bravo bajo condiciones de riego. De las líneas androestériles se sembraron cinco surcos de cinco metros de largo y 0,80m de separación y las demás líneas fueron sembradas en dos surcos a la misma distancia y longitud. Durante la época de floración en el mes de mayo se realizaron las cruzas entre las dos líneas androestériles y las cuatro restauradoras, así mismo se autopolinizaron las líneas B y R. Posteriormente se efectuaron muestreos de semilla tanto de las líneas como de los híbridos a los 0 , 11, 22 y 33 días después de alcanzar el grano la madurez fisiológica. Las muestras de semillas se enviaron inmediatamente al Laboratorio de Control de Calidad del Centro de Capacitación y Desarrollo de Tecnología de Semillas (CCDTS), de la Universidad Autónoma Agraria "Antonio Narro" en Buenavista, Saltillo, Coah. donde se determinó la calidad de las mismas.

En el laboratorio las líneas e híbridos se separaron para su análisis, se empleó en ambos casos un diseño de bloques completos al azar con arreglo factorial con 
Cuadro 1. Características agronómicas, de las líneas de sorgo seleccionadas para evaluar la calidad de la semilla. Tamaulipas, México, 1992-1993.

\begin{tabular}{lclll}
\hline Genealogía & \multicolumn{4}{c}{ Características } \\
\cline { 2 - 5 } & Tipo de línea & \multicolumn{1}{c}{ Color grano } & Color planta & Tipo de panoja \\
\hline LRB-104A & AyB & roJo & roJo & semicompacta \\
LRB-118A & AyB & rojo & canela & semicompacta \\
LRB-25 & $\mathrm{R}$ & crema & rojo & abierta \\
Tx-430R & $\mathrm{R}$ & crema & rojo & semicompacta \\
Tx-430C & $\mathrm{R}$ & crema & canela & semicompacta \\
SC-I03 & $\mathrm{R}$ & café & rojo & compacta \\
\hline
\end{tabular}

cuatro repeticiones. Las características evaluadas fueron: peso volumétrico (PV), peso de 1.000 semillas (PMS), vigor mediante la germinación normal después del envejecimiento acelerado (GNEA) y germinación standard normal (GSN).

Se realizó un análisis de varianza combinado para los híbridos y otro para las líneas. En las variables en donde se detectó significancia, se realizó una prueba de separación de medias por el método de Tukey en las líneas, mientras que dentro de híbridos se realizaron contrastes ortogonales. Para realizar los análisis de varianza se transformaron los datos del porcentaje de germinación normal y anormal por medio del arcoseno.

\section{RESULTADOS Y DISCUSIÓN}

\section{Calidad de la Semilla}

La semilla cosechada a madurez fisiológica tanto en líneas como en híbridos presentó una mayor calidad (Cuadro 2), medida ésta por un mayor valor en peso volumétrico (PV) y de 1.000 semillas, germinación standard y vigor (envejecimiento acelerado). Y esta calidad se redujo a medida que se atrasó la época de cosecha, lo cual coincide con lo indicado por Delouche, 1964.

Tomando como base los datos de una estación meteorológica distante $200 \mathrm{~m}$ del cultivo, durante el desarrollo del experimento las temperaturas máximas siempre estuvieron arriba de $30^{\circ} \mathrm{C}$ y las mínimas arriba de $23^{\circ} \mathrm{C}$. El porcentaje de humedad relativa máxima estuvo arriba del $75 \%$ y aunque esta medición coincidiócon la altura de la panoja del sorgo, se estima que estuvo más alta dentro del cultivo, dado la evaporación y transpiración de las plantas. La alta temperatura y humedad relativa fueron un factor propicio para la reducción de la calidad de la semilla después de la madurez fisiológica.
Cuadro 2. Calidad de la semilla de líneas e híbridos de sorgo cosechados en diferentes épocas. Campo Experimental Río Bravo. México. Otoño-invierno 1992-93.

\begin{tabular}{lccccc}
\hline $\begin{array}{l}\text { Días después } \\
\text { de Madurez }\end{array}$ & PV & GSN1 & PMS & GNEA \\
\multicolumn{1}{l}{ Fisiológica }
\end{tabular}

* Valores con la misma letra son estadísticamente no diferentes $(\mathrm{P} \leq 0,05)$.

${ }^{1}$ Los análisis de varianza fueron realizados con datos transformados por arcoseno.

C.V.= Coeficiente de Variación. P.V.= Peso Volumétrico. GSN= Germinación standard

GSEA= Germinación standard después del envejecimiento acelerado.

La presencia del viento durante la época de cosecha 22 y 23 días después de alcanzar el grano la madurez fisiológica, pudo haber contribuido a disminuir los efectos de deterioro de la calidad de la semilla, secando el entorno de éstas y disminuyendo los efectos de las altas temperatura y humedad relativa. 


\section{Líneas}

Uno de los criterios más antiguos y ampliamente usados para medir la calidad física de la semilla es el peso volumétrico (P.V.), el cual se define como el peso de grano con el cual se llena un volumen específico (Pushman; Bringham, 1975). En este estudio la línea LRB-118B en promedio presentó un mayor P.v. que la línea LRB-104B (Cuadro 3). Como estas líneas están muy emparentadas podemos aceberar que el P.v. de la LRB-118B fue menos afectado por el intemperismo. Respecto a las otras líneas se tiene que ser más cauteloso, dado que el P.V. al inicio es diferente para todos. Por lo tanto las inferencias no se deben de hacer respecto a un promedio sino respecto a la disminución que sufren a través del tiempo en que se atrasa la cosecha.

Cuadro 3. Peso volumétrico de seis líneas de sorgo cosechadas en diferentes épocas después de la madurez fisiológica. Tamaulipas, México. 1992-1993.

\begin{tabular}{lccccc}
\hline & \multicolumn{6}{c}{ Días después de madurez fisiológica } \\
\cline { 2 - 6 } Líneas & $\mathbf{0}$ & $\mathbf{1 1}$ & $\mathbf{2 2}$ & $\mathbf{3 3}$ & Promedio \\
\hline Tx-430C & 29,9 & 30,0 & 28,4 & 27,4 & $28,92 \mathrm{~b}$ \\
LRB-25 & 32,0 & 32,1 & 30,2 & 31,6 & $31,47 \mathrm{a}$ \\
SC-103 & 29,6 & 26,7 & 26,4 & 26,3 & $26,66 \mathrm{~b}$ \\
LRB-104B & 29,9 & 28,3 & 21,5 & 23,0 & $25,67 \mathrm{e}$ \\
LRB-118B & 31,8 & 30,8 & 28,8 & 24,4 & $28,95 \mathrm{~b}$ \\
Tx-430R & 33,0 & 31,4 & 31,0 & 31,5 & $31,72 \mathrm{a}$ \\
\hline
\end{tabular}

* Valores con la misma letra son estadísticamente no diferentes $(\mathrm{P} \leq 0,05)$

1 Los análisis de varianza fueron realizados con datos transformados por arcoseno.

De las líneas restauradoras la menos afectada por el intemperismo fue LRB-25. Esto puede estar relacionado al tipo de panoja que es abierta, se ventila más y es menos afectada por la humedad ambiental. Así mismo las líneas con mayor contenido de taninos fueron menos afectadas por el intemperismo (Tx-430R y SC-103 vs Tx-430C).

Respecto al peso de 1.000 semillas las líneas LRB104B y LRB-118B mostraron similares promedios, pero la primer línea presentó una reducción más grande cuando se le comparó con el peso de 1.000 semillas a madurez fisiológica (Cuadro 4). En este aspecto las líneas con mayor contenido de taninos no mostraron mayor peso de 1.000 semillas, dado que la línea Tx-430R tuvo un mayor decremento que Tx-430C y la línea SC103 presentó el mayor decremento de peso de 1.000 semillas. Esto es importante dado que el mayor contenido de taninos se ha asociado a una mayor resistencia al moho de grano. Los taninos pudieran estar evitando el desarrollo de hongos en el grano, pero no disminuyendo los procesos fisiológicos que reducen el peso de la semilla como son la lixiviación de minerales y la oxidación (Woodstock, et al. 1985). También debido a que SC-103 es de panoja compacta, pudiera estar siendo más afectada por intemperismo que los otros genotipos de panojas menos compactas.

En la germinación standard y vigor (Cuadros 5 y 6 ) se observó que la línea LRB-104B y LRB-118B presentaron valores muy similares, siendo mejor la línea LRB-104B en los dos primeros muestreos respecto a las líneas restauradoras, mientras que la línea LRB118B presentó un mayor vigor. Tx-430R presentó una mayor germinación standard pero un menor vigor que su contraparte isogénica Tx-430C. También se pudo observar que la línea con mayor contenido de taninos SC103 presentó la mayor germinación standard normal y el mayor vigor, en cambio la línea con menor contenido de taninos presentó la menor germinación standard y el menor vigor. $\mathrm{Al}$ respecto los taninos pudieron estar evitando el desarrollo de hongos, los cuales afectan la germinación y/o vigor de la semilla.

Cuadro 4. Peso de 1.000 semillas de seis líneas de sorgo sometidas a intemperismo. Tamaulipas, México. 1992-1993.

\begin{tabular}{lccccc}
\hline & \multicolumn{5}{c}{ Días después de madurez fisiológica } \\
\cline { 2 - 6 } Líneas & $\mathbf{0}$ & $\mathbf{1 1}$ & $\mathbf{2 2}$ & $\mathbf{3 3}$ & Promedio $^{1}$ \\
\hline Tx-430C & 31,8 & 31,5 & 28,8 & 25,8 & $29,5 \mathrm{~b}^{*}$ \\
LRB-25 & 28,7 & 27,3 & 30,7 & 27,7 & $28,6 \mathrm{c}$ \\
SC-103 & 36,5 & 27,2 & 32,8 & 26,4 & $30,7 \mathrm{a}$ \\
LRB-104B & 26,0 & 26,5 & 17,8 & 19,6 & $22,5 \mathrm{e}$ \\
LRB-118B & 24,7 & 22,4 & 22,1 & 20,5 & $22,4 \mathrm{e}$ \\
Tx-430R & 30,7 & 25,7 & 28,2 & 25,1 & $27,4 \mathrm{~d}$ \\
\hline
\end{tabular}

* Valores con la misma letra son estadísticamente no diferentes $(\mathrm{P} \leq 0,05)$.

${ }^{1}$ Los análisis de varianza fueron realizados con datos transformados por arcoseno.

\section{Híbridos}

En promedio la línea LRB-104A produjo híbridos con mayor $\mathrm{PV}$, aunque se pudo observar que los híbri dos con la línea LRB-118A presentaron un mayor peso 
Cuadro 5. Germinación normal standard en líneas de sorgo sometidas a intemperismo. Tamaulipas, México. 1992-1993.

\begin{tabular}{lccrcl}
\hline \multirow{2}{*}{ Líneas } & \multicolumn{6}{c}{ Días después de madurez fisiológica } \\
\cline { 2 - 6 } & $\mathbf{0}$ & $\mathbf{1 1}$ & $\mathbf{2 2}$ & $\mathbf{3 3}$ & Promedio \\
\hline Tx-430C & 33,8 & 22,5 & 54,3 & 26,3 & $34,2 \mathrm{e}^{*}$ \\
LRB-25 & 68,3 & 64,5 & 62,5 & 64,3 & $64,9 \mathrm{~b}$ \\
SC-103 & 81,0 & 73,8 & 83,3 & 76,8 & $78,7 \mathrm{a}$ \\
LRB-104B & 68,8 & 65,5 & 6,0 & 20,0 & $40,1 \mathrm{~d}$ \\
LRB-118B & 58,5 & 45,0 & 43,5 & 15,3 & $40,6 \mathrm{~d}$ \\
Tx-430R & 65,7 & 51,3 & 62,5 & 64,8 & $61,1 \mathrm{c}$ \\
\hline
\end{tabular}

* Valores con la misma letra son estadísticamente no diferentes $(\mathrm{P} \leq 0,05)$

1 Los análisis de varianza fueron realizados con datos transformados por arcoseno.

Cuadro 6. Germinación standard después del envejecimiento acelerado (vigor) en líneas de sorgo sometidas a intemperismo. Tamaulipas, México. 1992-1993.

\begin{tabular}{lccc}
\hline & \multicolumn{3}{c}{ Días después de madurez fisiológica } \\
\cline { 2 - 4 } Líneas & $\mathbf{0}$ & $\mathbf{1 1}$ & Promedio $^{1}$ \\
\hline Tx-430C & 58,0 & 54,0 & $56,0 \mathrm{c}^{*}$ \\
LRB-25 & 72,5 & 72,5 & $72,5 \mathrm{~b}$ \\
SC-I03 & 81,8 & 85,8 & $83,8 \mathrm{a}$ \\
LRB-I04B & 71,0 & 65,0 & $68,0 \mathrm{~b}$ \\
LRB-118B & 72,0 & 70,3 & $71,1 \mathrm{~b}$ \\
Tx-430R & 78,5 & 60,8 & $69,6 \mathrm{~b}$ \\
\hline
\end{tabular}

* Valores con la misma letra son estadísticamente no diferentes $(\mathrm{P} \leq 0,05)$

${ }^{1}$ Los análisis de varianza fueron realizados con datos transformados por arcoseno.

del primero al tercer muestreo, lo que confirma que dicha línea puede conferir una ventaja para esta característica a híbridos sometidos a intemperismo cuando la cosecha no se retrasa más de 20 días (Cuadro 7). El hecho de que características agronómicas favorables sean fácilmente heredadas a los híbridos por medio de alguno de sus progenitores, facilita grandemente la tarea del mejorador de plantas. Al respecto la susceptibilidad a la pérdida de peso volumétrico parece ser una característica varietal y el mejoramiento por selección bajo condiciones adecuadas puede ser posible (Czarnecki y Evans, 1986). El mayor PV juega un papel importante cuando el objetivo es el grano, en cambio cuando el objetivo es la semilla la germinación estándar y el vigor tienen prioridad.

En el peso de 1.000 semillas no se mostró una tendencia clara al comparar las hembras a través de los
Cuadro 7. Calidad de la semilla de sorgo a diferentes épocas de cosecha de híbridos con una hembra en común. Tamaulipas, México. 1992-1993.

\begin{tabular}{|c|c|c|c|c|c|}
\hline $\begin{array}{l}\text { Muestreo } \\
\text { D. a Mad. } \\
\end{array}$ & Hembra & P. V. & GSN(a) & P.M.S & GNEA $^{1}$ \\
\hline \multirow[t]{2}{*}{0} & LRB-104A & 31,207 & 86,54 & 25,93 & 88,0 \\
\hline & LRB-118A & $31,764 *$ & 86,63 & 29,75 & $89,0 *$ \\
\hline \multirow[t]{2}{*}{11} & LRB-I04A & 30,897 & 88,83 & 26,79 & 88,4 \\
\hline & LRB-118A & $31,(\mathrm{~J} 09 *$ & * $84,33 *$ & $25,48^{*}$ & $85,4^{*}$ \\
\hline \multirow[t]{2}{*}{22} & LRB-I04A & 30,656 & 88,81 & 25,42 & \\
\hline & LRB-118A & $30,779 *$ & $85,42 *$ & $26,04 *$ & \\
\hline \multirow[t]{2}{*}{33} & LRB-104A & 31,642 & 90,00 & 24,42 & \\
\hline & LRB-118A & $29,839 *$ & $81,90 *$ & $22,85^{*}$ & \\
\hline \multirow[t]{2}{*}{ Promedio } & LRB-I04A & 31,100 & 89,00 & 26,10 & 89,0 \\
\hline & LRB-118A & $30,700 *$ & $84,40 *$ & $25,00 *$ & $87,1 *$ \\
\hline
\end{tabular}

* Diferentes estadísticamente $(\mathrm{P} \leq 0,05)$ a LRB-1 04A.

${ }^{1}$ Los análisis de varianza fueron realizados con datos transformados por arcoseno.

P.V.=Peso volumétrico. GSN=Germinación estándar. PMS=Peso de 1.000 semillas. GNEA ${ }^{1}=$ Germinación estándar después del envejecimiento acelerado.

muestreos, siendo en promedio mejor los híbridos con la línea LRB-104A. La germinación standard fue mayor en los híbridos con la hembra LRB-104A a partir del segundo muestreo en donde la germinación en los híbridos con la hembra LRB-118A se redujo en una mayor proporción que en aquellos híbridos con la hembra LRB-104A. Respecto al vigor se presentó una tendencia similar. Demostrando que el PV es un parámetro de mayor importancia a evaluar para la calidad de semillas que el peso de 1.000 semillas.

La línea restauradora LRB-25 produjo los híbridos, con mayor peso volumétrico de grano, germinación estándar vigor y peso de 1.000 semillas. Esto puede explicarse por la mayor aptitud combinatoria general de la línea (Cuadro 8).

La mejor aptitud combinatoria específica para germinación estándar y vigor los presentó la cruza LRB104Ax LRB-25 (que corresponde al híbrido RB-4000), en PV fue para el híbrido LRB-118A x LRB-25 y en peso de 1000 semillas para la cruza LRB-104A x SC-103. (Cuadro 9).

La línea LRB-118B mostró mayor peso volumétrico y vigor que la línea LRB-104B, confIriéndose éstas ventajas a los híbridos formados con esta línea. En las características peso de 1.000 semillas y genninación estándar no se presentaron diferencias entre las líneas mantenedores, sin embargo los híbridos con LRB-104A mostraron mayores valores para estas características. 
Cuadro 8. Calidad de la semilla a diferentes épocas de cosecha de híbridos de sorgo con un progenitor restaurador en común. Tamaulipas, México. 1992-1993.

\begin{tabular}{lcccl}
\hline Machos & P.V. & GSN(a) & PMS & GNEA \\
\hline Tx-430C & 30,7 c* & $84,4 \mathrm{~b}$ & $24,7 \mathrm{c}$ & $88,3 \mathrm{a}$ \\
LRB-25 & $31,4 \mathrm{a}$ & $88,1 \mathrm{a}$ & $27,3 \mathrm{c}$ & $88,5 \mathrm{a}$ \\
SC-I03 & $31,0 \mathrm{~b}$ & $87,1 \mathrm{a}$ & $25,7 \mathrm{~b}$ & $87,8 \mathrm{ab}$ \\
Tx-430R & $30,5 \mathrm{c}$ & $87,1 \mathrm{a}$ & $24,4 \mathrm{c}$ & $87,1 \mathrm{~b}$ \\
\hline
\end{tabular}

* Valores con la misma letra son estadísticamente no diferentes $(\mathrm{P} \leq 0,05)$

1 Los análisis de varianza fueron realizados con datos transformados por arco seno.

P.V= Peso volumétrico GSN= Germinación estándar PMS = Peso de 1.000 semillas GNEA1= Germinadón estándar después del envejecimiento acelerado.

Cuadro 9. Calidad de la semilla de híbridos de sorgo cosechados en diferente época. Tamaulipas, México. 1992-1993.

\begin{tabular}{lllll}
\hline Machos & P.V. & GSN(a) & PMS & GNEA $^{1}$ \\
\hline Tx-430C x LRB-104A & 31,19 & 88,12 & 24,85 & 89,40 \\
LRB-118A & $30,15^{*}$ & $79,80^{*}$ & 24,60 & $9,41^{*}$ \\
LRB-25 x LRB-104A & 30,04 & 90,10 & 27,06 & 87,24 \\
LRB-118A & $31,75^{*}$ & $85,90^{*}$ & $27,60^{*}$ & $86,80^{*}$ \\
SC-103 x LRB-104A & 31,08 & 87,60 & 29,98 & 86,80 \\
LRB-118A & $30,79 *$ & $86,85^{*}$ & $24,46^{*}$ & $88,90^{*}$ \\
Tx-430R x LRB-104A & 30,82 & 88,47 & 25,39 & 88,00 \\
LRB-118A & $30,21 *$ & $85,96^{*}$ & $23,47 *$ & $86,30^{*}$ \\
\hline
\end{tabular}

* Diferente estadísticamente $(\mathrm{P} \leq 0,05)$ a híbridos con hembra LRB-104A.

1 Los análisis de varianza fueron realizados con datos transformados por arcoseno.

P.V.= Peso volumétrico GSN1= Germinación estándar PMS $=$ Peso de 1.000 semillas GNEA1= Germinación estándar después del envejecimiento acelerado.

La línea restauradora LRB-25 produjo los híbridos con mayor peso volumétrico del grano, germinación, vigor y peso de 1.000 semillas. Las variables P.V., PMS, GSN, GNEA son herramientas útiles en la selección y mejoramiento de los híbridos de sorgo en la selección contra el intemperismo.

\section{LITERATURA CITADA}

AVILA P., M.A.; ARELlANO, J. L. V; GÓMEZ, V A. J. 1994. Calidad de semillas de maíz durante la maduración. In XV Congreso de Fitogenética (Memorias) Monterrey, NL México. p.448.

BRAGONIER, H.W. 1976. Seed vigor-what is it? In: Seed vigor and deterioration. P.A. Bicentennial Symposium, Sponsored by the AOSA in Cooperation with Society of Commercial Seed Technologist and the ASTA. Pennsylvania USA. Butler, L.G. 1989. Sorghum polyphenols. In Toxicants of plant origino Vol. IV Phenolics, P.R. Cheeke, Ed. CRC Press, Inc., Boca Raton, FL. pp 95-121.

CZARNECKI E.; EVANS L. E. 1986. Effect of weathering during delayed harvest on test weight, seed size and grain hardness of wheat. Can. J. Plant Sci. 66: 473-482.

DELOUCHE, J.C. 1964. Seed maturation. Seed technology laboratory. Mississippi State University, prepared for International Training. Mississippi. U.S.A.

LÓPEZ S., M.; MOLINA M. J. C.; CARBALLO C. A. 1994. Deterioro de la germinación de semilla de cuatro especies en diferentes condiciones de almacenamiento. In: XV Congreso de Fitogenética (Memoria) Monterrey, N.L. México. p. 457.

PUSHMAN, F. M.; BRINGHAM, J. 1975. Components of test weight of ten varieties of winter wheat grown with two rates of nitrogen fertilizer application. J. Agric. Sci. 85: 559-563.

RAJANNA, B.; ANDREWS C. H. 1970. Trends in seed maturation of rice (Oryza sativa L.) Proc. AOSA. 60: 188-196.

RIVERA Z., L.; GASCA M. B. A. 1994. Efecto de cuatro fungicidas en la conservación de la calidad fisiológica en la semilla de maíz H-33. In: XV Congreso de Fitogenética (Memorias). Monterrey, N.L. México. p. 453.

ROBERTS, E.H. 1973. Predicting the storage life of seeds. Seed Sci. \& Technol. 1:499-514.

SARH, 1994. Estadísticas de producción del estado de Tamaulipas. p. 108.

WOODSTOCK, L.w.; FURMAN K.; LEFFLER H. R. 1985. Relationship between weathering deterioration and germination respiratory metabolism, and mineral leaching from cotton seeds. Crop. Sci. 25: 459-466. 